\title{
Equilibrium States of the Two-dimensional Ising Model in the Two-Phase Region
}

\author{
A. Messager and S. Miracle-Sole \\ Centre de Physique Théorique, C.N.R.S., Marseille, France
}

Received October 10, 1974

\begin{abstract}
We prove that at zero external field and for any temperature below the critical temperature, all translationally invariant equilibrium states for the two-dimensional Ising ferromagnet, are a convex combination of only two extremal states.
\end{abstract}

\section{Introduction}

A number of recent works deal with general properties of the equilibrium states of the Ising model. In particular, the following facts have been established. First, the Ising ferromagnet with an external field $h \neq 0$ has always a unique equilibrium state, as was proved by Ruelle [1] and Lebowitz and Martin-Löf [2]. It was also shown in [2] that there is only one equilibrium state at $h=0$ and above the critical temperature $T \geqq T_{c} ; T_{c}$ being defined as the temperature above which there is no spontaneous magnetization. For $h=0$ and at sufficiently low temperature $T \leqq T_{0}<T_{c}$, Gallavotti and Miracle-Sole [3] have shown that every translationally invariant equilibrium state is a convex linear combination of only two extremal states. This results hold in any dimension $v \geqq 2$. Moreover, in the two-dimensional case Lebowitz [4] has proved that the above defined value of $T_{c}$ coincides with the Onsager value of the critical temperature.

It is the aim of this paper to show that in the two-dimensional case, for $h=0$ and for all values of the temperature below the critical value $T_{c}$, every translationally invariant equilibrium state is a convex linear combination of only two extremal equilibrium states. The description of the translationally invariant equilibrium states of the two-dimensional Ising ferromagnet is then complete. This model has two pure phases coexisting when $h=0$ and $T<T_{c}$, and only one pure phase is present for all other values of $h$ and $T$.

We recall that, following Dobrushin [5] and Lanford and Ruelle [6], an equilibrium state of the infinite system may be defined as a family of correlation functions $\left\langle\sigma_{a_{1}} \ldots \sigma_{a_{n}}\right\rangle$ for the finite sets $\left\{\sigma_{a_{1}}, \ldots, \sigma_{a_{n}}\right\}$ of spins of the lattice, obtained as the limit of correlation functions for a sequence of finite boxes with some boundary conditions. The state is translationally invariant if $\left\langle\sigma_{a_{1}+a} \ldots \sigma_{a_{n}+a}\right\rangle=\left\langle\sigma_{a_{1}} \ldots \sigma_{a_{n}}\right\rangle$ for all $a$ of the 
lattice. Let us denote by $\left\langle\sigma_{a_{1}} \ldots \sigma_{a_{n}}\right\rangle^{+}$and $\left\langle\sigma_{a_{1}} \ldots \sigma_{a_{n}}\right\rangle^{-}$the infinite volume correlation functions when all spins of the boundary are fixed to be +1 and -1 respectively. Our statement can be precised in the following form.

Theorem. If $h=0$ and $T<T_{c}$, any translationally invariant equilibrium state $\left\langle\sigma_{a_{1}} \ldots \sigma_{a_{n}}\right\rangle_{0, T}$ of the two-dimensional Ising ferromagnet (with nearest neighbour interaction), is such that

$$
\left\langle\sigma_{a_{1}} \ldots \sigma_{a_{n}}\right\rangle_{0, T}=\alpha\left\langle\sigma_{a_{1}} \ldots \sigma_{a_{n}}\right\rangle_{0, T}^{+}+(1-\alpha)\left\langle\sigma_{a_{1}} \ldots \sigma_{a_{n}}\right\rangle_{0, T}^{-}
$$

for some $0 \leqq \alpha \leqq 1$.

The states $\left\langle\sigma_{a_{1}} \ldots \sigma_{a_{n}}\right\rangle^{+}$and $\left\langle\sigma_{a_{1}} \ldots \sigma_{a_{n}}\right\rangle^{-}$which can be called the up-magnetized and down-magnetized phases, have very special physical properties [7], justifying their character of pure phases. They are translationally invariant, verify a cluster property, and are extremal in the set of all equilibrium states at given $h$ and $T$.

These two states are related simply by

$$
\left\langle\sigma_{a_{1}} \ldots \sigma_{a_{n}}\right\rangle^{+}=(-1)^{n}\left\langle\sigma_{a_{1}} \ldots \sigma_{a_{n}}\right\rangle^{-}
$$

They are different in the region covered by the theorem, as follows from the equality

$$
m_{T}=\left\langle\sigma_{a}\right\rangle_{0, T}^{+}
$$

where $m_{T}$ is the Onsager-Yang value of the spontaneous magnetization, and the fact that $m_{T}$ is strictly positive when $T<T_{c}$. This last equality has been proved by Benettin, Gallavotti, Jona-Lasinio, and Stella [8], but it is itself a consequence of the theorem. The two states coincide when $h \neq 0$ or when $T \geqq T_{c}$ since, as we have already mentioned, the equilibrium state is then unique.

Let us point-out for completeness certain known facts about the behaviour of the pure phases. First (see [1]), in the region $h \neq 0$ the correlation functions $\left\langle\sigma_{a_{1}} \ldots \sigma_{a_{n}}\right\rangle_{h, T}$ are analytic functions of $h$ and $T$, and

$$
\left\langle\sigma_{a_{1}} \ldots \sigma_{a_{n}}\right\rangle_{0, T}^{ \pm}=\lim _{h \rightarrow 0^{ \pm}}\left\langle\sigma_{a_{1}} \ldots \sigma_{a_{n}}\right\rangle_{h, T}
$$

Secondly, when $h=0$, the correlation functions $\left\langle\sigma_{a_{1}} \ldots \sigma_{a_{n}}\right\rangle_{0, T}^{+}$and $\left\langle\sigma_{a_{1}} \ldots \sigma_{a_{n}}\right\rangle_{0, T}^{-}$are known to be infinitely differentiable with respect to $T$, as was proved by Gallavotti and Lebowitz [9].

Finally, it has been conjectured that the two-dimensional Ising ferromagnet has no non-translationally invariant equilibrium states, even in the region $h=0, T<T_{c}$; while in the case of three or more dimensions one knows from Dobrushin [10], that such states exist at low temperature. We, unfortunately, will not be able to illuminate this problem. 
The idea of our proof is to use, as in [8], the duality transformation, and to apply then some known results about the low activity expansions. We introduce a small even perturbation, such as $\lambda \Sigma_{a} \sigma_{a_{1}+a} \ldots \sigma_{a_{n}+a}$, in the hamiltonian. Because the equilibrium state is unique above the critical temperature $T_{c}$, it will follow that the perturbed thermodynamic pressure has a derivative with respect to $\lambda$ at $\lambda=0$. The same fact can be proved below $T_{c}$ by constructing the dual perturbed pressure. This will allow us to conclude at the unicity of the even correlation functions. This statement, as we will see, is equivalent to the theorem. We can finally remark that the exact calculations, existing in the literature (see for instance [11]), of the even correlation functions at $h=0$ are then justified to be independent on the boundary condition.

\section{Formulation of the Problem}

We consider a two-dimensional square lattice $\mathscr{L}$. We call a geometric figure built with a certain number of bonds of the lattice a closed polygon if at every lattice point only an even number (zero, two or four) of bonds occurs. It is clear that to every configuration of +1 and -1 spins in the middle of the squares of the lattice can be associated a closed polygon in the following way. A bond belongs to the polygon if it separates different spins and does not belong to the polygon if it separates equal spins. The same closed polygon is associated to two symmetric configurations, in which the +1 and -1 spins are interchanged.

Let us fix a finite square region $\Lambda$ of the lattice. It is well known that the partition function of the two-dimensional Ising model (with only nearest neighbour interactions) at zero magnetic field can easily be written as

$$
Z_{\Lambda}(T)=\sum_{n \geqq 0} C_{n}(\Lambda) e^{-\frac{2 J}{k T} n}
$$

where $C_{n}(\Lambda)$ is the number of $n$-sided closed polygons that can be drawn inside $\Lambda$. The thermodynamic free energy, or the pressure in the language of lattice gases, is given by

$$
p(T)=\lim _{\Lambda \rightarrow \infty} \frac{1}{|\Lambda|} \log Z_{\Lambda}(T)
$$

where $|\Lambda|$ is the number of points of $\Lambda$, and it is known to be independent on the boundary conditions.

It will be useful to write the partition function in the following way

$$
Z_{Q}(z)=\sum_{X \subset Q} C(X) z^{|X|}
$$

where $z=\exp \left(-\frac{2 J}{k T}\right), Q$ represents the set of bonds of the lattice inside $A, X$ is a subset of this set (we will call it a configuration of bonds), $|X|$ the number of elements of $X$, and $C(X)=1$ if $X$ is a closed polygon and $C(X)=0$ otherwise. We remark that the factor $C(X)$ appears as 
coming from a four-body potential of a hard-core interaction between bonds obliging them to occur in an even number at every vertex.

Consider now the set of correlation functions associated to the equilibrium states. We shall restrict ourselves to the even correlation functions $\left\langle\sigma_{a_{1}} \ldots \sigma_{a_{2 n}}\right\rangle$. It is clear that all of them will be well determined if we know the expectation values $\left\langle\sigma_{x_{1}} \ldots \sigma_{x_{n}}\right\rangle$ of the products of spin variables associated with the bonds: $\sigma_{x}(X)=-1$ if $x \in X, \sigma_{x}(X)=+1$ if $x \notin X$. Consider for instance the two spin correlation function $\left\langle\sigma_{a_{1}} \sigma_{a_{2}}\right\rangle$. If $\Gamma$ is an arbitrary path connecting the two sites $a_{1}$ and $a_{2}$, and $x_{1}, x_{2}, \ldots, x_{n}$ are the bonds cut by this path, one has the path independent relation

$$
\left\langle\sigma_{a_{1}} \sigma_{a_{2}}\right\rangle=\left\langle\sigma_{x_{1}} \ldots \sigma_{x_{n}}\right\rangle .
$$

The above relation can be generalized to any correlation function containing an even number of points, $2 n$, by dividing them into $n$ pairs and by considering paths connecting the two points of each pair.

In order to study these correlation functions we introduce the following perturbed pressure

where

$$
p(z, \lambda)=\lim _{\Lambda \rightarrow \infty} \frac{1}{|\Lambda|} \log Z_{Q}(z, \lambda)
$$

$$
Z_{Q}(z, \lambda)=\sum_{X \subset Q} C(X) z^{|X|} e^{-\lambda \sum_{x \in Q} \sigma_{M+x}(X)}
$$

and $M$ is a fixed finite set of bonds. We have denoted by $M+x$ the translated of $M$ by the vector characterizing the bond $x$, by $Q_{1}$ the set of $x \in Q$ such that $M+x \subset Q$, and by $\sigma_{S}(X)$ the product

$$
\sigma_{S}(X)=\prod_{x \in S} \sigma_{x}(X)=(-1)^{|X \cap S|} .
$$

The correlation functions $\left\langle\sigma_{T}\right\rangle$ for a finite volume depend on the boundary condition we choose. It is known, however, (see for instance [1]), that the translationally invariant correlation functions for the infinite system will be unique, if and only if the perturbed pressure $p(z, \lambda)$ has a derivative at the point $\lambda=0$, for any set $M$ of bonds. This is the point we are going to consider in the following.

\section{Duality Applied to the Perturbed Pressure}

Next we will apply the duality transformation to the perturbed pressure. We first recall the following relations among the bond spin variables

$$
\begin{aligned}
\sigma_{X}(Y) & =\sigma_{Y}(X) \\
\sigma_{X}(Y, Z) & =\sigma_{X}(Y) \sigma_{X}(Z) \quad \text { where } \quad Y, Z=Y \cup Z \backslash Y \cap Z \\
\sum_{X \subset Q} \sigma_{Y}(X) \sigma_{Z}(X) & =0 \quad \text { if } \quad Y \neq Z ; \quad=2^{|Q|} \quad \text { if } \quad Y=Z ; \quad Y, Z \subset Q .
\end{aligned}
$$


These relations are easily established and they are well known in the formalism of classical spin systems. We remark that

$$
C(X)=\prod_{a \in \mathscr{L}} \frac{(-1)^{\left|Q_{a} \cap X\right|}+1}{2}=\prod_{a \in \mathscr{L}} \frac{\sigma_{Q_{a}}(X)+1}{2}
$$

where the product extends to all vertex of the lattice and $Q_{a}$ denotes the set of the four bonds coming to the vertex $a$. This expression follows from the definition of a closed polygon. We have then

$$
C(X)=2^{-|\mathscr{L}|} \sum_{A \subset \mathscr{L}} \sigma_{\tilde{A}}(X)
$$

where to every $A \subset \mathscr{L}$ we associate the set $\tilde{A}=\Pi_{a \in A} Q_{a}$.

Let us represent the bonds of the lattice by their middle points and remember that $C(X)=1$ means that $X$ is a closed polygon of the lattice. It is easily seen that the set $\tilde{A}$ is in fact a closed polygon in the dual lattice represented in the figure by the dotted lines cf. the figure. We shall write then $C^{\prime}(\hat{A})=1$, and in general $C^{\prime}(Y)=1$ to say that $Y$ is a closed polygon in the dual lattice. This allows us to write

$$
C(X)=2^{-|\mathscr{L}|} \sum_{Y \subset Q} C^{\prime}(Y) \sigma_{Y}(X) .
$$

From this we deduce that

hwere $z^{*}$ is given by

$$
\begin{aligned}
\sum_{X \subset Q} C(X) z^{|X|} & =2^{-|\mathscr{L}|} \sum_{X \subset Q} \sum_{Y \subset Q} C^{\prime}(Y) \sigma_{Y}(X) z^{|X|} \\
& =2^{-|\mathscr{L}|+|Q|}(1+z)^{-|Q|} \sum_{Y \subset Q} C^{\prime}(Y) z^{*|Y|}
\end{aligned}
$$

$$
z^{*}=\frac{1-z}{1+z}
$$

and corresponds to the dual temperature. The same calculation shows that

$$
\begin{aligned}
\sum_{X \subset Q} C(X) z^{|X|} \sigma_{T}(X) & =2^{-|\mathscr{L}|+|Q|}(1+z)^{-|Q|} \sum_{Y \subset Q} C^{\prime}(Y) z^{*|Y . T|} \\
& =2^{-|\mathscr{L}|+|Q|}(1+z)^{-|Q|} \sum_{Y \subset Q} C^{\prime}(Y) z^{*|Y|}\left(z^{*}\right)^{\sum_{y \in T} \sigma_{y}(Y)} .
\end{aligned}
$$

This formulae lead to the known expressions [8] for the dual unperturbed pressure and the dual correlation functions. As we see in the figure the original lattice corresponds to the closed boundary condition (all spins equal to +1 or -1 in the boundary), while the dual lattice corresponds to the free boundary condition (all spins free in the boundary).

For the perturbed partition function we find

$$
\begin{aligned}
Z_{Q}(z, \lambda) & =\sum_{X \subset Q} C(X) z^{|X|} e^{-\lambda \sum_{x \in Q_{1}} \sigma_{M+x}(X)} \\
& =(\operatorname{ch} \lambda)^{\left|Q_{1}\right|} \sum_{X \subset Q} C(X) z^{|X|} \prod_{x \in Q_{1}}\left(1+\tau \sigma_{M+x}(X)\right) \\
& =(\operatorname{ch} \lambda)^{\left|Q_{1}\right|} \sum_{X \subset Q} C(X) z^{|X|} \sum_{S \subset Q_{1}} \tau^{|S|} \sigma_{M(S)}(X)
\end{aligned}
$$


where $\tau=\operatorname{th} \lambda$ and $M(S)=\Pi_{x \in S}(M+x)$. And by using the precedent formulae

with

$$
Z_{Q}(z, \lambda)=2^{-|\mathscr{L}|+|Q|}(1+z)^{-|Q|}(\operatorname{ch} \lambda)^{\left|Q_{1}\right|} Z_{Q}^{\prime}\left(z^{*}, \lambda\right)
$$

$$
Z_{Q}^{\prime}\left(z^{*}, \lambda\right)=\sum_{X \subset Q} C^{\prime}(X) z^{*|X|} \sum_{S \subset Q_{1}} \tau^{|S|}\left(z^{*}\right)^{\sum \in M(S)} \sigma_{y}(X) .
$$

We arrive then, at the following expression

$$
p(z, \lambda)=C_{0}+p_{1}\left(z^{*}, \tau\right)
$$

where $p_{1}\left(z^{*}, \tau\right)$ is the thermodynamic pressure corresponding to the partition function $Z_{Q}^{\prime}\left(z^{*}, \lambda\right)$. The term $c_{0}=2 \log \frac{\sqrt{2} \operatorname{ch} \lambda}{1+z}$ will be unimportant in the following.

\section{Derivability of the Perturbed Pressure}

We first consider the expression

$$
\left.\sum_{S \subset Q_{1}} \tau^{|S|} z^{*}\right)^{\sum^{\sum} \sum_{(S)} \sigma_{y}(X)}=\sum_{S \subset Q_{1}} \tau^{|S|} e^{-U_{X}(S)}
$$

appearing at the end of the preceding section. We have written

$$
U_{X}(S)=-\left(\log z^{*}\right) \sum_{y \in M(S)} \sigma_{y}(X)
$$

to indicate that $U_{X}(S)$, for a fixed $X$, can be regarded as a potential energy for the configuration $S$. In fact

where

$$
U_{X}(S)=\sum_{R \subset X} \Phi_{X}(R)
$$

$$
\Phi_{X}(R)=-\left(\log z^{*}\right) 2^{|R|}(-1)^{|S|-|R|} \sum_{\substack{y \in \cap(M+x) \\ x \in R}} \sigma_{y}(X)
$$

and we see that $U_{X}(S)$ comes from a non-translationally invariant many-body potential, which has the important property of having a finite range. That is, $\Phi_{X}(R)=0$ if the diameter of the set $R$ is larger than a fixed distance, corresponding to the fact that then the intersection of all the sets $M+x$ is empty. This fixed distance is independent of $X$. Moreover the following norm

$$
\left\|\Phi_{X}\right\|=\sup _{x} \sum_{R \ni x}\left|\Phi_{X}(R)\right|
$$

can be majorized by a number which is independent on $X$.

The expression that we are considering can be written as

$$
\begin{aligned}
\sum_{S \subset Q_{1}} \tau^{|S|} e^{-U_{X}(S)} & =\exp \left\{\int_{0}^{\tau} \frac{d t}{t} \frac{\sum_{S \subset Q_{1}} t^{|S|}|S| e^{-U_{X}(S)}}{\sum_{S \subset Q_{1}} t^{|S|} e^{-U_{X}(S)}}\right\} \\
& =\exp \left\{\sum_{x \in Q_{1}} \int_{0}^{\tau} \frac{d t}{t} \varrho_{X, Q_{1}, t}(x)\right\}
\end{aligned}
$$


where we have used the notation

$$
\varrho_{X, Q_{1}, t}(x)=\frac{\sum_{x \in S \subset Q_{1}} t^{|S|} e^{-U_{X}(S)}}{\sum_{S \subset Q_{1}} t^{|S|} e^{-U_{X}(S)}}
$$

to indicate that $\varrho_{X, Q_{1}, t}(x)$ is the density at the point $x$ of a certain system in a box $Q_{1}$, with interaction energy $U_{X}(S)$ and activity $t$.

We collect now a certain number of results concerning this density at small activity. We refer for them to [12] and we remark that the results there remain valid for non-translationally invariant potentials, provided that the norm $\left\|\Phi_{X}\right\|$ of the interaction is finite. We denote by $\left\|\mathscr{K}_{\Phi_{x}}\right\|$ the norm of the kernel that appears in [12] and we guarantee by taking an $r_{0}>0$ small enough that $\mid \mathscr{K}_{\Phi_{x}} \| \leqq B<1$ when $|t| \leqq r_{0}$. Because $\left\|\Phi_{X}\right\|$ can be uniformly bounded, $r_{0}$ and $B$ can be chosen independently of $X$. The results we shall use are the following

a) $\left|\varrho_{X, Q_{1}, t}(x)\right| \leqq|t|\left(1-\left\|\mathscr{K}_{\Phi_{X}}\right\|\right)^{-1} \leqq|t|(1-B)^{-1}$,

b) the following limits

$$
\lim _{Q_{1} \rightarrow \infty} \varrho_{X, Q_{1}, t}(x)=\varrho_{X, t}(x)
$$

exist, and

$$
\left|\varrho_{X, Q_{1}, t}(x)-\varrho_{X, t}(x)\right|<f(\delta)
$$

where $f(\delta)$ is a function of the distance $\delta$ from $X$ to the boundary of $Q_{1}$ which is such that $f(\delta) \rightarrow 0$ when $\delta \rightarrow \infty$.

c) $P_{X, t}(x)$ is an analytic function of $t$ in the circle $|t| \leqq r_{0}$.

Let us denote by $Q$ a square of side equal to $L$. We shall first prove that

$$
\begin{aligned}
p_{1}\left(z^{*}, \tau\right)= & \lim _{L \rightarrow \infty} \frac{1}{L^{2}} \log \sum_{X \subset Q} C^{\prime}(X) z^{*|X|} \exp \left\{\sum_{x \in Q} \int_{0}^{\tau} \frac{d t}{t} \varrho_{X, Q_{1}, t}(x)\right\} \\
& \lim _{L \rightarrow \infty} \frac{1}{L^{2}} \log \sum_{X \subset Q} C^{\prime}(X) z^{*|X|} \exp \left\{\sum_{x \in Q} \int_{0}^{\tau} \frac{d t}{t} \varrho_{X, t}(x)\right\} .
\end{aligned}
$$

In fact, the difference between the two expressions, before taking the limit, can be bounded by

$$
\frac{1}{L^{2}}\left\{4 L(\log L)|\tau|(1-B)^{-1}+L^{2} f(\log L)\right\} .
$$

The first term comes from the estimate (a) and takes into account the contribution of the points $x$ which are at a distance of the boundary of $Q_{1}$ less than $\log L$. The second term comes from the estimate (b) and takes into account the difference between the two densities at the other points of $Q_{1}$. By taking the limit when $L \rightarrow \infty$ one gets the result.

The second step will consist in introducing a new pressure

$$
p_{2}\left(z^{*}, \tau\right)=\lim _{L \rightarrow \infty} \frac{1}{L^{2}} \sum_{X \subset Q} C^{\prime}(X) z^{*|X|} \exp \left\{\tau \sum_{x \in Q}\left(z^{*}\right)^{\sum_{y \in M+x} \sigma_{y}(X)}\right\}
$$


and proving that

$$
\lim _{\tau \rightarrow 0}\left|\frac{p_{1}\left(z^{*}, \tau\right)-p_{1}\left(z^{*}, 0\right)}{\tau}-\frac{p_{2}\left(z^{*}, \tau\right)-p_{2}\left(z^{*}, 0\right)}{\tau}\right|=0 .
$$

In order to prove this result, we first remark that

$$
\left.\frac{d \varrho_{X, t}(x)}{d t}\right|_{t=0}=\left(z^{*}\right)^{\sum \sum_{y+x} \sigma_{y}(X)}
$$

as follows from a direct calculation. Because we know by (c), that $\varrho_{X, t}(x)$ is an analytic function of $t$ for $|t| \leqq r_{0}$, we can write

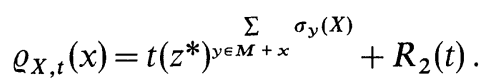

Taking (a) into account we bound the remainder $R_{2}(t)$ of the Taylor series by

Then

$$
\left|R_{2}(t)\right| \leqq \frac{|t|^{2}}{r_{0}^{2}} \frac{r_{0}(1-B)^{-1} r_{0}}{r_{0}-|t|}
$$

$$
\left|p_{1}\left(z^{*}, \tau\right)-p_{2}\left(z^{*}, \tau\right)\right| \leqq \frac{1}{L^{2}}\left|\sum_{x \in Q} \int_{0}^{\tau} \frac{d t}{t} R_{2}(t)\right| \leqq \frac{2|\tau|^{2}(1-B)^{-1}}{2\left(r_{0}-|\tau|\right)}
$$

and this second result follows.

Remembering now the duality relation between $p$ and $p_{1}$ we conclude at the following statement.

Lemma. The perturbed pressure $p(z, \lambda)$ has a derivative with respect to $\lambda$ at the point $\lambda=0$, if and only if, the same is true for $p_{2}\left(z^{*}, \lambda\right)$.

In fact the two derivatives coincide, since $\lambda$ and $\tau=$ th $\lambda$ differ only by a term of second order in $\lambda$. This gives the following dual relation between the translationally invariant correlation functions

$$
\left\langle\sigma_{M}\right\rangle_{z}=\left\langle\left(z^{*}\right)^{\sum \in M} \sigma_{y}\right\rangle_{z^{*}}
$$

This relation could be deduced from Section III for particular boundary conditions.

\section{Proof of the Theorem}

We have already mentioned that the existence of the derivative of the perturbed pressure $p(z, \lambda)$ with respect to $\lambda$ at $\lambda=0$ is equivalent to the unicity of the translationally invariant even correlation functions for the infinite system. Now, it is well known that $z=z^{*}$ at the critical temperature $T_{c}$, i.e. when $z=z_{c}$. On the other hand, when $z$ varies in the interval $\left[0, z_{c}\right], z^{*}$ varies in the interval $\left[z_{c}, 1\right]$. We know also from Lebowitz [4], that the equilibrium state is unique in the interval $\left[z_{c}, 1\right]$, corresponding to $T \geqq T_{c}$. Therefore, $p_{2}\left(z^{*}, \lambda\right)$, which is also a perturbed pressure for the Ising model, has a derivative with respect to $\lambda$ at $\lambda=0$ for $z_{c} \leqq z \leqq 1$. By applying the lemma of the preceding section we deduce 
that also $p(z, \lambda)$ has such a derivative, for $z$ in the interval $\left[0, z_{c}\right]$, i.e. for $T$ between 0 and $T_{c}$. Hence the translationally invariant even correlation functions are also unique in this interval. It remains only to show that this last statement is equivalent to the main theorem that we have formulated in the Introduction, to conclude our proof.

This equivalence follows from a known argument ${ }^{1}$ based one the fact that the translationally invariant equilibrium states form a Choquet simplex. Let us suppose that $\left\langle\sigma_{a_{1}} \ldots \sigma_{a_{n}}\right\rangle$ is an extremal invariant equilibrium state. Then $\frac{1}{2}\left\langle\sigma_{a_{1}} \ldots \sigma_{a_{n}}\right\rangle+\frac{1}{2}(-1)^{n}\left\langle\sigma_{a_{1}} \ldots \sigma_{a_{n}}\right\rangle$ is also an invariant equilibrium state. It coincides with $\frac{1}{2}\left\langle\sigma_{a_{1}} \ldots \sigma_{a_{n}}\right\rangle^{+}+\frac{1}{2}\left\langle\sigma_{a_{1}} \ldots \sigma_{a_{n}}\right\rangle^{-}$, because the two are equal by hypothesis when $n$ is even, and are zero when $n$ is odd. But the decomposition of this state in extremal states has to be unique in a Choquet simplex. Therefore, only the two states $\left\langle\sigma_{a_{1}} \ldots \sigma_{a_{n}}\right\rangle^{+}$and $\left\langle\sigma_{a_{1}} \ldots \sigma_{a_{n}}\right\rangle^{-}$are extremal in the set of the invariant equilibrium states, and the theorem is proved.

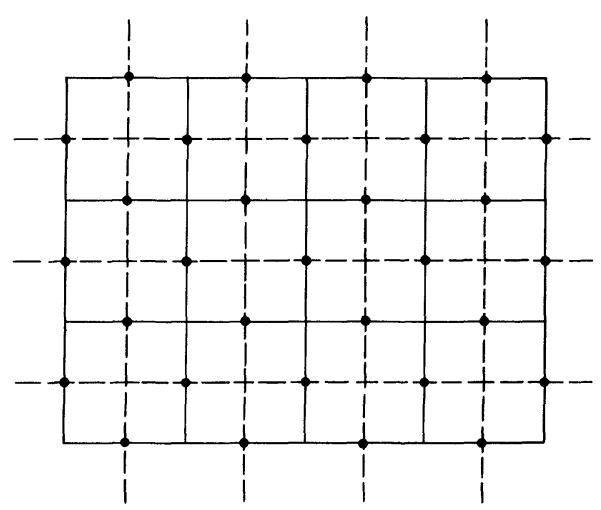

Acknowledgements. It is a pleasure to thank J. L. Lebowitz for helpful discussions.

\section{References}

1. Ruelle, D.: Ann. Phys. 69, $364-374$ (1972)

2. Lebowitz, J., Martin-Löf, A. : Commun. math. Phys. 25, 276-282 (1972)

3. Gallavotti, G., Miracle-Sole, S.: Phys. Rev. 5 B, 2555-2559 (1972)

4. Lebowitz, J. L.: Commun. math. Phys. 28, 313 (1972)

5. Dobrushin, R. L.: Teoriya Veroyatnostli ill Primeneniya 13, $201-229$ (1968)

6. Lanford, O., Ruelle, D. : Commun. math. Phys. 13, 194-215 (1969)

7. Gallavotti, G.: Riv. Nuovo Cimenti 2-2, 133-169 (1972)

8. Benettin, G., Gallavotti, G., Jona-Lasinio, G., Stella, A.L.: Commun. math. Phys. 30, 45-54 (1973)

9. Gallavotti, G., Lebowitz, J.L.: Physica 70, 219-221 (1973)

10. Dobrushin, R.L.: Gibbsian states describing the co-existence of phases in the threedimensional Ising model. Probability Theory and its Applications (to appear)

\footnotetext{
${ }^{1}$ Communicated to us by Ruelle.
} 
11. McCoy, B., Wu, T.: The two-dimensional Ising model. Cambridge (Mass.): Harvard University press 1973

12. Gallavotti, G., Miracle-Sole, S.: Commun. math. Phys. 7, 274 -288 (1968)

Communicated by G. Gallavotti

\author{
A. Messager \\ S. Miracle-Sole \\ Centre de Physique Théorique \\ C.N.R.S. \\ 31, Chemin J. Aiguier \\ F-13274 Marseille Cedex 2, France
}

